\title{
Assessment of Multi-Criteria Preference Measurement Methods for a Dynamic Environment
}

\author{
Annika Lenz \\ University of Hohenheim, Information Systems I, \\ Stuttgart, Germany \\ annika.lenz@uni-hohenheim.de
}

\author{
Mareike Schoop \\ University of Hohenheim, Information Systems I, \\ Stuttgart, Germany \\ schoop@uni-hohenheim.de
}

\begin{abstract}
Multi-criteria decision analysis is required in various domains where decision making reoccurs as part of a longer-term process. When the decision context changes or the preferences evolve due to process dynamics, one-shot preference measurement is not sufficient to build an adequate basis for decision making. Process dynamics require taking into account the dimension of time. We investigate six interactive preference measurement methods providing the possibility to assess alternatives in terms of utility for an individual decision maker, whether they are suitable for dynamic preference adjustment. We use a mixed-methods approach to analyse them towards 1) requirements for a dynamic method, and 2) their efficiency, validity, and complexity. Our results show that the best method to be further developed for dynamic context is Adaptive Self-Explication slightly preferable over Pre-Sorted Self-Explication. Our assessment implicates that an extension of the Adaptive Self-Explication will enable efficient dynamic decision support.
\end{abstract}

\section{Introduction}

Decision making with multiple objectives is required in a wide range of domains. To support such multi-criteria decision making, a plethora of methods for measuring the decision maker's preferences have been developed. The aim of preference measurement methods is to assess feasible alternatives for the decision maker to support his/her decision making. Usually, preference measurement methods assume a static context [3]. Such approaches require the decision maker to provide all preference information at the time of measurement. In other words, a one-shot preference measurement is performed without considering changes.

However, in many cases, the decision maker does not have complete knowledge at the time when preference measurement is necessary. Dynamic decision making problems with multiple, conflicting objectives appear in many real-world scenarios [20], e.g. optimising traffic [31], replacements configurations for multi-component systems [6], surgical patients' prioritisation [1], or decision support in negotiations [24]. In the following, we explain preference changes in a dynamic context using the example of negotiations. In negotiations a variety of decisions must be made. To have an adequate basis for decision making, i.e. articulate, assess, and compare offers, preferences must be measured very early, in fact prior to the actual negotiation phase.

Negotiation processes are shaped and influenced by their dynamic nature. They are interdependent processes between different parties, which implies that a negotiator is influenced by and must rely on his/her partner. Thus, they are in a two-way process that does not give them sole control. This reciprocity means that the parties must exchange views and information in order to reach a joint solution. The exchange process takes place iteratively and builds on the previous exchange. This way both partners gain new information during the process.

This new information in turn influences the shape of the negotiation itself. For example, parties might find out or wish for themselves that they should negotiate more issues than they had previously assumed. This means, the scope of the negotiation and therewith the decision problem is changed. By the change of the scope, i.e. introducing new attributes, new alternatives, or withdrawing attributes or alternatives, preferences articulated at the beginning of a negotiation are obsolete. Furthermore, whilst maintaining the same agenda throughout the negotiation, preferences might change simply by being provided with new information, having more clarity in the process, or by process dynamics $[8,43]$ according to dissonance theory [13] and reactance theory [5]. Dissonance theory proposes that negotiators perceive the value of their outcome, i.e. the offer chosen, higher in retrospective [8, 43], while reactance theory 
postulates that alternatives are perceived more attractive if they are jeopardised to be forfeited and vice versa $[8,43]$. This means that even if information is not outdated, negotiators may desire to readjust their preferences due to the negotiation process dynamics. In general, preference formation, learning or fatigue may cause preference dynamics [33].

To sum up, by change of scope, increase of information level, or process dynamics, preferences change. As outlined in the example, the need for dynamic, interactive methods becomes clear [20, 31]. However, such dynamic, interactive approaches are still scarce, since these methods must take a new dimension into account: time [3, 31]. The considered decision problems are time-dependent as soon as new knowledge affects the decisional context: attributes, alternatives, or preference information. In many practical situations it is unachievable to provide preference information for all decision elements involved beforehand [3]. New methods try to take incomplete or imprecise preference information into account [e.g. 18, 35, 36]. However, literature on interactive, dynamic methods is still scarce [31].

Our aim is to investigate to what extent interactive, multi-criteria preference measurement approaches are suitable in a dynamic environment. We analyse the methods' potential to be extended to allow for dynamic preference adjustment. Helping a decision maker to fine-tune his/her preferences in an environment where the decision context itself changes over time, is challenging, especially when a high number of objectives is involved [31]. We, therefore, follow a three-step approach to reach this aim:

RQ1: What are the requirements for individual interactive dynamic preference measurement?

RQ2: Do preference measurement methods address the requirements identified?

RQ3: What potential do preference measurement methods have to be expanded to fit dynamic demands?

We hereby focus on preference measurement for an individual decision maker. The method must allow interactivity [cf. 31], to allow the decision maker to redefine his/her preferences according to an increasing knowledge.

To answer our research questions, we derive general requirements for such a method and select methods, which are supposed to meet the identified requirements. To assess the selected methods, we follow a mixed-methods approach, which both qualitatively evaluates the fulfilment of the identified requirements as well as their expandability to handle preference adjustments and quantitatively evaluates their performance regarding common preference measurement criteria, i.e. validity, efficiency, and complexity. Both the qualitative and quantitative assessment are utilised to select the best suitable method to be extended by dynamic means.

To this end, this paper is structured as follows. In section 2, we outline theoretical background on preference measurement methods and related work on dynamic preference measurement. Section 3 identifies relevant requirements for dynamic preference measurement and selects promising methods. The selected methods are assessed by their fulfilment of the identified requirements and evaluated in section 4 . Section 5 concludes this paper and gives a brief outlook.

\section{Theoretical Background and Related Work}

A multitude of approaches exists to assess alternatives respectively choose the 'best' alternative. In this chapter, we review general approaches based on multi-attribute utility theory [23] and preference measurement methods, which take a temporal perspective.

\subsection{Utility Based Multi-Criteria Decision Analysis Approaches}

Multi-criteria decision analysis methods comprise of a plethora of approaches. Apart from outranking methods (e.g. the ELECTRE family [37] or PROMETHEE family [4]), fuzzy methods, multiobjective optimisation, robust ordinal regression methods [15], and UTA methods [21], we focus on approaches, which provide the possibility to assess all alternatives in a multi-criteria decision problem in terms of utility applying multi-attribute utility theory [23].

In concordance with multi-attribute utility theory, different utility functions can be applied [14]. We concentrate on a linear-additive utility function by which the utility of a chosen set of alternatives is calculated by the sum of utility values $u$ for an alternative $x_{i}$ of the attribute $i$ weighted by the relative importance $\mathrm{w}_{\mathrm{i}}$ of the attribute $\mathrm{i}$.

$$
U(A)=\sum_{i} w_{i} * u\left(x_{i}\right)
$$

A rough categorisation of utility based methods, which allow assessment of all alternatives mainly driven by marketing research is the division into decompositional (conjoint analyses [17, 19]), compositional (self-explicated approaches, AHP [25, 38]), and hybrid approaches [16].

Conjoint analysis estimates the customer's preferences, "given his or her overall evaluations of a set of alternatives that are prespecified in terms of 
levels of different attributes" [17, p. 4]. While a disadvantage of conjoint analysis has been that it results in an information overload for the respondent using a large number of attributes [17], newer methods and its applications have overcome this shortcoming [cf. 33].

The self-explicated approach is a compositional approach [17], which questions the respondent separately on each attribute (stage 1) and on the attribute importance weights (stage 2). Thereby, it minimises the information-overload problem [41]. Hence, the traditional self-explicated approach can handle a large number of attributes and levels [33].

Hybrid approaches have been developed aiming to combine the benefits of conjoint measurement and self-explicated methods [17, 41]. They comprise of both a self-explicated stage and a decompositional stage taking results of the previous stage into account and/or calibrating resulting preferences from both stages [2].

\subsection{Dynamic Multi-Criteria Decision Analysis Methods}

Current research streams for time-dependent preference measurement comprise of methods, which allow preference adjustment without changing objectives and alternatives of the decision problem, evolutionary dynamic multi-objective optimisation using big data, dynamic adjustment of feasible alternatives, and periodic interactive verification.

Reiser [35] develops an approach, which considers incomplete and uncertain information at the time of preference measurement. He suggests a two-part approach. As long as information is incomplete he uses Fast Polyhedral Adaptive Conjoint Estimation (FastPACE) [42] to gradually complete preference information. At the point the information level has increased to a sufficient degree, he applies ex post preference measurement using Adaptive SelfExplication (ASE) [32]. Reiser [35] focuses on incomplete and uncertain information of importance weightings and preferences for alternatives. The scope of the decision problem remains the same throughout the process.

In evolutionary dynamic multi-objective optimisation, the goal is to "find the set of trade-off solutions that is as close as possible to the set of optimal trade-off solutions" [20, p. 1256]. Nebro et al. [31] develop an interactive approach in dynamic context for multi-objective optimisation. They use evolutionary algorithms based on reference points, which can be modified interactively, to handle changes in the environment. The overall goal is to solve an optimisation problem by the best suitable solution.

Certa et al. [6] aim to dynamically support a decision maker in the domain of replacement configurations for multi-component systems. Dynamic updates capture changes of information about the decisional context. Their goal, too, is to find an optimal solution. They use a two-step approach comprising of obtaining the set of non-dominated trade-off solutions and comparing them to select the best one. These steps are repeated sequentially to include information about the decision problem. ELECTRE III [37] is used to select the best solution at the time required.

Abbasgholizadeh Rahimi et al. [1] develop a dynamic framework, considering risks and uncertainties in the context of surgical patients' prioritisation. The dynamic aspect is that patients are added and removed from waiting lists as well as their condition evolves over time, which needs to be evaluated. Their three-step framework covers defining the decision problem structure and attributes, defining and evaluating alternatives and finally a dynamic evaluation of the alternatives on a periodic update basis, while the objectives and importance weightings remain the same.

Benítez et al. [3] use AHP [38] extended by the time dimension. Calculation of importance weighting is based on pairwise comparisons. Their focus is on ensuring the consistency of information gathered by the pairwise comparisons. Preference information is allowed to be provided at several times.

Roszkowska and Wachowicz consider preference measurement respectively decision support for illdefined decision problems in negotiations [36]. They apply fuzzy TOPSIS to an ill-structured negotiation problem. Their aim is to maintain a stable preference model, which is based on the aspiration level and reservation level only, throughout the negotiation process. They do not consider preference changes, but focus on taking new offers into account, which lie outside of the initially defined negotiation space. They suggest solutions to evaluate such out-of-space offers without affecting the evaluation of prior exchanged offers. In such a case, the fuzzy TOPSIS algorithm is applied without involvement of the decision maker.

In contrast, DeSarbo et al. [10] and Liechty et al. [28] focus on preference changes respectively preference adjustment due to several factors such as learning, exposure to additional information, fatigue, and cognitive limitations. They develop a Bayesian dynamic linear methodology to capture dynamic adjustment processes, which allows for heterogeneous level estimation. 
Guo et al. [18] consider agents to learn the negotiator's preferences to be applied in multiattribute negotiations. They develop an algorithm, which enables agents to learn their users' preference structures over time and build a multi-attribute utility function.

To this end, dynamic preference measurement methods which cover big data approaches [e.g. 31], evolutionary algorithms [e.g. 18, 31], methods, which solve optimisation problems [e.g. 31, 6], or use outranking approaches [e.g. 6], have been proposed.

Since it is very difficult and complex to measure preferences dynamically [31], new methods for this purpose focus on certain aspects of the dynamic decisional context. Of the interactive ones for an individual decision maker, which provide the possibility of assessing all alternatives in terms of utility, dynamic methods focus either on changes of the decision scope [e.g. 36] without preference changes, alternative and alternative assessment changes [e.g. 1], preference development [e.g. 35], or focus on consistency issues of additional paired comparisons [e.g. 3].

\section{Selection of an Adequate Preference Measurement Method}

In this chapter, we identify requirements for an individual, interactive dynamic preference measurement method and develop an evaluation scale. Moreover, we select six methods, which will be analysed towards their suitability in a dynamic environment.

\subsection{Requirements and Assessment Scale}

We derive the following requirements for a preference measurement method to capture a dynamic environment. The benefit of dynamic preference measurement is to enable dynamic decision support, which is usually provided for an individual decision maker [cf. 34]. As the analysis of a set of attributes is an individual's task, or the task of a homogeneous group of stakeholders [e.g. 11], individual preference measurement is required. Thus, the method must be able to estimate preferences for an individual decision maker (R1).

The focus of this paper is to evaluate preference measurement methods by their suitability for dynamic preference measurement. Therefore, the preference measurement method must be able to handle changes in the scope of the decision problem (R2), i.e. changes of attributes and alternatives [cf. 12]. It must handle modification of attributes and alternatives, namely efficient adding and withdrawing must be possible.
Along with the change of scope, preferences for additional elements must be complemented without distorting existing preferences and if possible, without the necessity to measure all preferences again. Equally, deleting objectives or attributes must not affect valid preferences for the remaining objectives and attributes (R3).

Moreover, if the scope of the decision problem remains the same throughout the process, preferences regarding the objectives or attributes may change nonetheless [cf. 43]. Hence, the preference measurement method must be able to adjust the initially elicited preferences efficiently (R4).

The methods are assessed, if they fulfil ('+') or do not fulfil ('-') the requirements R1-R4. If the methods do not meet the specified requirements sufficiently, we will evaluate them on a scale from '--' to ' ++ ', how well they can be expanded to meet the requirements, i.e. allow efficient individual dynamic preference adjustment. A negative assessment means that the method cannot be expanded to adjust preferences dynamically, neutral assessment means that an expansion for dynamic preference adjustment is possible, but the preference adjustment requires high effort, while positive assessment means that the method can be expanded for efficient dynamic preference adjustment. We differentiate between two levels in both the negative and the positive range to indicate the effort required for dynamic preference adjustment.

\subsection{Selection of Methods}

To meet the requirements, we elicit state-of-the-art preference measurement methods, which allow individual preference measurement. Self-explicated approaches are well suited to measure preferences for multi-attribute products that involve a large number of attributes [32, 39]. In recent years, new self-explicated methods have been suggested, which include an adaptive question design to reduce effort for the decision maker [35].

ASE by Netzer and Srinivasan [32] is a newer compositional preference measurement approach with adaptive question design. It is designed to solve the self-explicated constant sum question problem when the number of product attributes becomes large [32]. The preferences for alternatives are elicited by rating them on a defined rating scale. The relative attribute importance weightings are elicited by first ranking the attributes according to their importance and second comparing pairs of attributes. The pairwise comparisons are chosen adaptively based on their potential to provide the most information. 
An extension of ASE is the Presorted Adaptive Self-Explicated Approach (PASE) by Schlereth et al. [39]. It is also a self-explicated approach of adaptive character. Additionally to ASE, it proposes to rate the attributes according to their importance prior to the attribute ranking in order to presort them. They argue that positioning a task, which is not trade-off based, simplifies the subsequent trade-off based task. A presorted list of attributes is supposed to reduce the cognitive burden in the ranking task.

The Paired Comparison-based Preference Measurement (PCPM) by Scholz et al. [40] is an alternative compositional approach using constantsum paired comparison questions. PCPM [40] utilises AHP to estimate part worths, but reduces the set of pairwise comparisons. The overall decision problem is divided into subproblems on different levels, for which preferences are elicited. The question design aims to compare each attribute (alternative) with four other attributes (alternatives) to reduce the number of comparisons. Missing preference relations are calculated based on known comparison information.

It is argued that new self-explicated approaches are superior over conjoint analyses regarding a high number of attributes, usability for respondents or ease in terms of data collection [e.g. 29]. However, to gain comprehensive insights, we include the Conjoint Adaptive Ranking Database (CARDS) by Dahan [9] as a newer conjoint analysis, which follows an adaptive design. The main concept of CARDS builds on two ideas [7]: to measure the decision maker's elimination process by identifying his/her simplification decision rule and thus consider only key attributes; and to avoid inconsistent answers by guiding the decision maker towards consistent answers. During the process, inconsistent product combinations are eliminated as soon as they are classified as the same.

Hybrid methods are designed to combine the best of self-explicated and conjoint approaches. The Adaptive Conjoint Analysis (ACA) [2, 22] is an adaptive method developed by Johnson and enhanced by Sawtooth Software, Inc. It follows a hybrid approach and enjoys large popularity among researchers and practitioners [30]. In the compositional stage, both the desirability of alternatives [2] and the relative attribute importance across attributes are rated. Self-explicated part worths are calculated [32]. The decompositional stage builds on results of the compositional stage. Pairedcomparison trade-off questions are asked to compare up to five attributes updating the utility estimates [2]. The paired-comparison questions are adaptively chosen based on estimated utility, the question design, and the frequency of attributes compared. The resulting estimates of both stages are combined based on a weighting factor.

FastPACE by Toubia et al. [42] is a state-of-the-art hybrid method with adaptive comparison tasks at an individual level. It is built on ACA with the aim of an efficient design of the adaption of new stimuli. The aim is to reduce the number of product combinations by polyhedral question design. It combines the selfexplicated approach with conjoint analysis by graded paired comparisons of partial product profiles, which are chosen adaptively [32].

To this end, the methods we will evaluate in this paper cover three self-explicated approaches (ASE, PASE, PCPM), one conjoint analysis (CARDS), and two hybrid approaches (FastPACE, ACA).

\section{Assessment of Selected Methods}

In this chapter, we will assess the selected methods using a mixed-methods approach. In section 4.1., we will qualitatively analyse, if these methods address the requirements, respectively how well they can be expanded to fulfil them. The methods are quantitatively analysed towards their performance, i.e. efficiency, validity, and difficulty, in section 4.2.

\subsection{Qualitative Fulfilment of Requirements}

In the following, we analyse the selected methods towards their fulfilment of the identified requirements. ASE, PASE, PCPM, CARDS, FastPACE and ACA all are applicable for an individual decision maker (R1). None of the methods requires preferences of other respondents with similar preference profiles.

The requirement to handle changes in the scope of the decision problem (R2) is not addressed by the methods. None of them stipulates to add or withdraw objectives or attributes.

Since none of the methods stipulates attribute or value changes, preference elicitation of new attributes and values respectively preference adjustment of remaining attributes and values (R3) is not considered.

Regarding preference adjustment due to preference changes independently of attribute and alternative changes (e.g. due to an increasing information level, R4), CARDS does not stipulate preference changes. Beyond that, it does not use inconsistent answers to review elicited preferences, but to reduce the response error by deleting inconsistent response options [7]. In PCPM, adjustments of preferences are stipulated neither. PCPM utilises a fixed question design for comparisons, which does neither suggest how to determine comparisons in case of new/obsolete attributes/alternatives nor does it provide possibilities for additional comparisons in case of preference 
changes. In ACA and FastPACE, update mechanisms for part worths exist. This is done by conducting new set(s) of comparisons. After each set of comparisons, the part worths are updated. However, a deletion of obsolete preferences is not stipulated, so the initial preferences would be considered as well. Regarding ASE and PASE new comparisons can be conducted, if preference changes occur. The estimation of the part worths is done after each set of comparison taking all information into account. So in case of changes, the calculation can be applied as it is provided in ASE already. However likewise as in ACA and FastPACE and as in all one-shot methods, deletion of outdated information is not stipulated.

Regarding the possibility to expand the methods to fit dynamic demands, CARDS focuses on key attributes, which is contradictory to the aim of an expansion, because it is supposed to assess all attributes. The main concepts of CARDS is to eliminate less important attributes and inconsistent response options. Thus, if changes force the addition or deletion of attributes or alternatives, the method must be conducted from scratch. In PCPM, expansion is possible in principle. However because of the fixed question design, changes require very high effort. Even deletion of attributes/alternatives requires to conduct new comparisons and entails a number of recalculations. In ACA, scaling in stage 1 must be repeated based on the original scores. In stage 2, additional comparisons must be determined and asked. The mechanism to update preference information is of limited usage, since in case of deletion, outdated information must be deleted and thus the calculation of utility estimated redone from scratch. Moreover, because of rescaling in stage 1 , the combination of estimates of stage 1 and stage 2 must be recalculated. Since FastPACE builds on ACA, but focuses on the improvement of the question design, the expansion is similar to ACA.

Expansion of ASE and PASE is possible. The single steps rating of alternative desirabilities, rating of attributes (in case of PASE), and ranking of attributes can be repeated for new attributes and/or alternatives. Subsequently, new attributes and alternatives (in case of new best or worst alternatives) require new attribute comparisons. In case of attribute or alternative deletion, the respective comparison information can be deleted from the elicited preferences. Deletion may also result in new attribute comparisons. If attributes and values remain the same, but their preferences change, the elicited preference information may be deleted, and consequently the elicitation steps for single attributes or alternatives repeated as well as new attribute comparisons conducted. In each case, the part worths may be estimated after the respective set of attribute comparisons utilising all secured preference information.

Table 1 gives an overview of the assessment of the methods to evaluate. The methods differ in the possibility of an efficient expansion. Since CARDS does not show potential to be expanded, it will be excluded from further consideration. Both ASE and PASE show the most potential for expansion.

Table 1. Assessment of requirements. *) New information can be considered during the elicitation.

\begin{tabular}{|c|c|c|c|c|c|}
\hline \multirow{2}{*}{ Method } & \multicolumn{4}{|c|}{ Fulfilment of requirements } & \multirow{2}{*}{$\begin{array}{c}\text { Possibility } \\
\text { to expand }\end{array}$} \\
\cline { 2 - 5 } & $R 1$ & $R 2$ & $R 3$ & $R 4$ & \\
\hline ASE & + & - & - & $\left.+^{*}\right)$ & ++ \\
\hline PASE & + & - & - & $+*)$ & ++ \\
\hline PCPM & + & - & - & - & $\circ$ \\
\hline ACA & + & - & - & $+*)$ & + \\
\hline FastPACE & + & - & - & $+*)$ & + \\
\hline CARDS & + & - & - & - & -- \\
\hline
\end{tabular}

\subsection{Quantiative Performance Assessment}

In the following, the selected methods are evaluated based on validity (convergent validity, discriminant validity, and predictive validity), efficiency, and complexity to ensure that the best suitable methods do not suffer major shortcomings regarding state-of-the-art performance criteria.

CARDS was shown to be not suitable for efficient expansion by dynamic means. Hence, in the following only ACA, FastPACE, ASE, PASE, and PCPM are assessed. For this purpose, we discuss recent studies by Schlereth et al. [39], who evaluate ASE, PASE, and PCPM; Meißner et al. [30], who evaluate ACA, ASE, and PCPM; and Netzer and Srinivasan [32], who evaluate ACA, FastPACE, and ASE.

We assess the methods in relation to each other according to the empirical evaluation in the original studies. Thus, we use a relative scale from '--' to ' ++ ', negative/positive range meaning worse/better than other method(s) evaluated in the respective measures.

4.2.1. Validity. Regarding convergent validity, Schlereth et al. report high correlations for the attribute importance weights across ASE and PASE (>0.75, PCPM n.a.) in their first survey and also high correlations for ASE, PCPM, and PASE (>0.65) in their second survey [39]. In line with these results, Meißner et al. report high correlations of ACA and ASE (0.84), ACA and PCPM (0.84), and ASE and PCPM (0.89) [30]. Thus, high convergent validity is assumed for all evaluated methods. 
Discriminant validity is measured between attribute importance weights by the range between most and least important attributes at a measurement method level. ASE, PASE, and PCPM show the best discriminant validity among all of their evaluated methods [39]. Discriminant validity across respondents (measured by average dispersion across respondents) is reported for ASE (Mean Std. in first survey $=9.07$, Mean Std. in second survey =9.66) and PASE (Mean Std. in first survey $=9.43$, Mean Std. in second survey $=9.50$ ) as very high, and PCPM still high (first survey n.a., Mean Std. in second survey $=$ 5.96) [39]. Discriminant validity across individuals for ASE and PCPM is reported as much higher than for ACA [30], measured by the dispersion of the attribute importance weights. Moreover, the variance of the attribute importance weights is significantly different for only one case between ASE and PCPM, while it is different between ACA and PCPM for 12 cases and between ACA and ASE for 11 cases, which means that the divergent importance of attributes is captured better by ASE and PCPM [30].

Predictive validity is measured differently in the empirical studies applied. Schlereth et al. [39] measure predictive validity (1) by hit rates of predicting the first-choice of three alternatives in four holdout tasks; (2) perceived quality of importance weights, for which respondents rated how well the importance weights determined matched their true importance weights; (3) hit rate for the identification of own importance weights among the set of four alternative importance weights. (1) shows low significant superiority of PASE compared to ASE in their first survey, however, no significant difference of PASE, ASE, and PCPM in their second survey. (2) shows significant difference of PASE compared to ASE in their first survey, however no significant difference of ASE, PASE, and PCPM in their second survey. (3) shows low significant difference of PASE compared to ASE, and high significant difference of PCPM to ASE, while there is no significant difference of PCPM and PASE. Netzer and Srinivasan [32] measure individual-level predictive validity by (1) the hit rates of predicting the highest-ranked alternative in each of the two validation sets (choice set hit rate); (2) the hit rate for the 12 pairwise choices derived from the ranking of four alternatives in their two choice sets; (3) average rank-order correlation between predicted and actual ranking for each respondent. Each three measures show that ACA and FastPACE have significantly lower predictive validity than ASE. Meißner et al. [30] report no significant differences between their evaluated methods for the hit rate of the best alternative, however, report a low significant difference of PCPM compared to ACA for the hit rate of the ranking of alternatives.

4.2.2. Efficiency. Mixed results are reported regarding the effort a respondent expends on the preference measurement tasks. ASE is significantly shorter than PASE in Schlereth et al.'s first survey except for the ranking task, however, no significant differences are reported for their second survey [39]. The survey duration using PCPM is significantly shorter than using ASE and PASE [39]. Meißner et al. [30] report an average duration of $6.51 \mathrm{mins}$ for PCPM, $8.10 \mathrm{mins}$ for ASE, and 12.78 mins for ACA, while Netzer and Srinivasan [32] report ACA as fastet (14.45 mins), followed by ASE (15.10 mins) and FastPACE (21.60 mins). They assume a delay of six to eight seconds between the paired comparison questions resulting from using interpreted code to be responsible for the significant longer duration of FastPACE.

However, Schlereth et al. [39] argue that if methods stay below the mark of 20 mins, the effort associated is unlikely overwhelming for respondents. Therefore, all evaluated methods except for FastPACE, probably due to implementation issues, are well suited.

Table 2. Validity, efficiency, and difficulty of the selected methods [30, 32, 39]

\begin{tabular}{|c|c|c|c|c|c|}
\hline 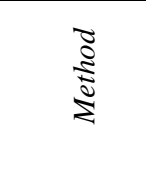 & 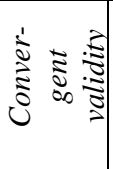 & 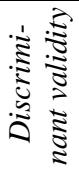 & 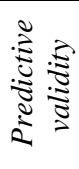 & 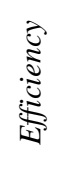 & $\underset{\substack{0 \\
:}}{\stackrel{0}{5}}$ \\
\hline ACA & ++ & $\circ$ & $\circ$ & \begin{tabular}{l|l}
$\circ$ & + \\
\end{tabular} & \begin{tabular}{l|l}
${ }^{\circ}$ & + \\
\end{tabular} \\
\hline FastPACE & & & o & - & o \\
\hline$\overline{\mathrm{ASE}}$ & ++ & + & $\begin{array}{l}{ }^{\circ} \mid+ \\
\mid++\end{array}$ & + & $\begin{array}{c}{ }^{\circ}|+| \\
++\end{array}$ \\
\hline PASE & ++ & + & + & o & + \\
\hline PCPM & + & $\begin{array}{r}+1 \\
++ \\
\end{array}$ & $\begin{array}{l}+1 \\
++ \\
\end{array}$ & ++ & $\begin{array}{l}+1 \\
++ \\
\end{array}$ \\
\hline
\end{tabular}

4.2.3. Survey difficulty. Survey complexity of ASE and PASE is considered equally high, but differs significantly to PCPM, which is perceived easier than ASE and PASE [39]. Contrary, Meißner et al. [30] report for task difficulty that ASE is significantly better than ACA and PCPM. Regarding ASE, ACA, and FastPACE no significant differences in difficulty are reported.

However, all methods - where ratings are reported - are perceived as rather of low complexity, because ASE, PASE, and PCPM are evaluated on an average > 4 at a scale from 1 "very high perceived task complexity" to 7 "very low perceived task complexity" [39] and ACA, ASE, and PCPM are rated 
6.63 and above on a 9-item scale from 1 "very difficult" to 9 "very easy" [30].

Table 2 gives an overview of the discussed measures. None of the methods shows clear superiority. FastPACE shows weaknesses regarding efficiency. However, none of the methods is assessed as not suitable.

\subsection{Discussion and Evaluation}

Studies argue that hybrid methods are most suitable for the class of multi-criteria decision problems considered (e.g. preference measurement in negotiations with incomplete information [35]). Our comparison - without focus on incomplete information but well considering increasing information - cannot support this argument, since ACA as hybrid method and ASE as a self-explicated method both fulfil the requirement of preference adjustment and common validity and efficiency criteria. Moreover, ASE performs better regarding validity and efficiency [30].

The long-standing critique of self-explicated approaches that they do not capture trade-offs [17] is addressed by Netzer and Srinivasan [32]. They break down the attribute importance rating into an attribute ranking task followed by pairwise comparisons of attributes, which are appropriate in practical situations [3].

Furthermore, self-explicated approaches are criticised that it is difficult to allocate a constant sum across a large number of attributes. ASE, PASE and PCPM alleviate this problem by ranking the attributes and/or conducting pairwise comparisons, in which the decision maker distributes a constant sum among the two attributes to be compared [32, 39, 40].

Regarding self-explicated approaches, it is argued, that the first stage of self-explicated approaches, in which the desirability of alternatives is rated directly, is perceived as complex and results in high cognitive burden. However, in two-stage self-explicated approaches, irrespectively of the particular method, the first stage is considered to provide reliable results with good predictive power [39].

Self-explicated approaches are well suited to measure consumer preferences for multi-attribute products that involve many attributes [32]. Since decompositional approaches become taxing with more attributes, which results in higher effort and cognitive burden for the decision maker [39], self-explicated approaches are currently more popular to estimate preferences for complex products [39, 40].

Regarding the qualitative and the quantitative assessment, we found a self-explicated method to be superior to hybrid methods, namely the ASE approach by Netzer and Srinivasan [32] (cf. Table 3). In line with our finding, Matzner et al. [29] choose a selfexplicated approach over conjoint analysis for their preference measurement in information systems design choices, because of the superior handling of a large number of attributes, greater usability for respondents, and greater ease in terms of data collection.

Table 3. Overall evaluation of the preselected methods. H: Hybrid; C: Conjoint analysis; SE: Self-Explicated.

\begin{tabular}{|c|c|c|c|c|c|c|}
\hline \multirow[b]{2}{*}{ 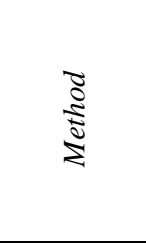 } & \multirow[b]{2}{*}{$\stackrel{8}{2}$} & \multicolumn{2}{|c|}{$\begin{array}{l}\text { Qualitative } \\
\text { assessment }\end{array}$} & \multicolumn{3}{|c|}{$\begin{array}{l}\text { Quantitative } \\
\text { assessment }\end{array}$} \\
\hline & & 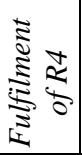 & 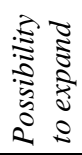 & $\frac{\vec{D}}{i=0}$ & 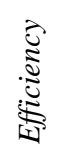 & $\underset{0}{\stackrel{\Xi}{\Xi}}$ \\
\hline $\mathrm{ACA}$ & $\mathrm{H}$ & $+*)$ & + & + & \begin{tabular}{l|l}
$\circ$ & + \\
\end{tabular} & \begin{tabular}{l|l}
$\circ$ & +
\end{tabular} \\
\hline FastPACE & $\mathrm{H}$ & $\left.t^{*}\right)$ & + & o & - & $\circ$ \\
\hline CARDS & $\mathrm{C}$ & - & -- & & & \\
\hline ASE & $\mathrm{SE}$ & $+*)$ & ++ & ++ & + & + \\
\hline PASE & $\mathrm{SE}$ & $\left.t^{*}\right)$ & ++ & ++ & o & + \\
\hline PCPM & $\mathrm{SE}$ & - & $\circ$ & ++ & ++ & $\begin{array}{l}++ \\
+\end{array}$ \\
\hline
\end{tabular}

\section{Conclusion and Outlook}

Individual interactive preference measurement in a dynamic environment is challenging [31]. We evaluated self-explicated approaches - ASE, PASE, and PCPM - a conjoing analysis approach - CARDS - and hybrid approaches - ACA and FastPACE using a mixed-methods approach. Their suitability for a decision context, in which the decision problem itself may change over time, is both qualitatively (if the methods fulfil the identified requirements respectively if the can be expanded to fulfil them), and qunatiatively assessed (validity, efficiency, difficulty). Our result is that none of the methods is suitable in a dynamic environment per se, however, some methods provide inherent mechanisms, which can be reused for preference adjustment processes. ASE, PASE, ACA, and FastPACE provide direct ratings and paired comparisons, which can be repeated. Recalulation of the utility model is required, however, these methods do already update utility values after sets of comparisons. ASE and PASE as self-explicated approaches have the advantage that attribute and value preferences are separated per se (although they are connected because attributes are assessed based on their best and worst cases), which simplifies repetition of single preference questions, while an efficient dynamic extension of ACA and FastPACE is more 
complex, because the conjoint part builds upon the direct ratings. Hence, repetition of steps of the compositional and the decompositional approach would be necessary, which results in higher effort.

Regarding validity, efficiency, and difficulty of the survey, ASE and PASE are superior to ACA and FastPACE. ASE outperforms PASE in terms of efficiency, because PASE includes an additional step of rating attributes according to their importance. The value of this step, which is intended to reduce the cognitive burden on the decision makers, however, is not great enough to be reflected in the difficulty.

The aim of this study cannot be to provide a holistic study comparing all existing preference measurement methods. We aimed to consider the most appropriate, most promising state-of-the-art methods. However, we cannot ensure that a well-suitable one was missed.

We did not evaluate the performance of the methods by ourselves but refered to empirical studies, which limits the comparability of the empirical results. However, our attempt to remedy this limitation was to involve three different studies to gain a comprehensive picture.

The results of this paper are generalisable to all contexts, in which an individual decision maker must measure preferences for a multiple objective decision problem applying multi-attribute utility theory where the decision problem scope and/or preferences change over time to require the decision maker to interactively adjust his/her preferences. Such circumstances are found in various application domains, e.g. optimising traffic [31], replacements configurations for multicomponent systems [6], surgical patients' prioritisation [1], or decision support in negotiations [24], e.g. requirements negotiations [27].

Our results suggest to extend ASE for a dynamic context. As in our introductory example of preference measurement in negotiations, we have developed a dynamic preference adjustment method based on ASE to fit dynamic demands for a negotiation context and designed dynamic decision support for the case of requirements negotiations [26].

\section{Acknowledgements}

We gratefully acknowledge A. Lenz' PhD grant by the State Baden-Württemberg and the support of the research area "Negotiation Research - Transformation, Technology, Media, and Costs", Faculty of Business, Economics, and Social Sciences at the University of Hohenheim.

\section{References}

[1] Abbasgholizadeh Rahimi, S., A. Jamshidi, A. Ruiz, and D. Ait-kadi, "A new dynamic integrated framework for surgical patients' prioritization considering risks and uncertainties", Decision Support Systems, 88, 2016, pp. $112-120$.

[2] https://www.sawtoothsoftware.com/support/technicalpapers/aca-related-papers/aca-technical-paper-2007, accessed 3-9-2017.

[3] Benítez, J., X. Delgado-Galván, J. Izquierdo, and R. Pérez-García, "An approach to AHP decision in a dynamic context", Decision Support Systems, 53(3), 2012, pp. 499 506 .

[4] Brans, J.P. and P. Vincke, "Note-A Preference Ranking Organisation Method", Management Science, 31(6), 1985, pp. 647-656.

[5] Brehm, S.S. and J.W. Brehm, Psychological reactance: A theory of freedom and control, Academic Press, New York, 1981.

[6] Certa, A., M. Enea, and T. Lupo, "ELECTRE III to dynamically support the decision maker about the periodic replacements configurations for a multi-component system", Decision Support Systems, 55(1), 2013, pp. 126134.

[7] http://www.anderson.ucla.edu/faculty/ely.dahan/content /EXPLOR_Award_Winner.ppt, accessed 5-26-2017.

[8] Curhan, J.R., M.A. Neale, and L. Ross, "Dynamic valuation: Preference changes in the context of face-to-face negotiation", Journal of Experimental Social Psychology, 40(2), 2004, pp. 142-151.

[9] Dahan, E., Conjoint adaptive ranking database system: Working paper, 2007.

[10] DeSarbo, W.S., D.K.H. Fong, J. Liechty, and J.C. Coupland, "Evolutionary preference/utility functions: A dynamic perspective", Psychometrika, 70(1), 2005, pp. 179-202.

[11] Felfernig, A., C. Zehentner, G. Ninaus, H. Grabner, W. Maalej, D. Pagano, L. Weninger, and F. Reinfrank, "Group Decision Support for Requirements Negotiation", in Advances in User Modeling, Springer, Berlin Heidelberg, 2012.

[12] Fernandes, M., Agenda negotiations in electronic negotiation support systems: An information systems perspective, Books on Demand, Norderstedt, 2016.

[13] Festinger, L., A theory of cognitive dissonance, Stanford University Press, Stanford, Calif., 1957.

[14] French, S., J. Maule, and N. Papamichail, Decision behaviour, analysis and support, Cambridge University Press, Cambridge, UK, New York, 2009.

[15] Greco, S., V. Mousseau, and R. Słowiński, "Ordinal regression revisited: Multiple criteria ranking using a set of 
additive value functions", European Journal of Operational Research, 191(2), 2008, pp. 416-436.

[16] Green, P.E., "Hybrid Models for Conjoint Analysis: An Expository Review", Journal of Marketing Research, 21(2), 1984, p. 155.

[17] Green, P.E. and V. Srinivasan, "Conjoint Analysis in Marketing: New Developments with Implications for Research and Practice", Journal of Marketing, 54(4), 1990, p. 3.

[18] Guo, Y., J.P. Müller, and C. Weinhardt, "Learning User Preferences for Multi-attribute Negotiation: An Evolutionary Approach", in Multi-Agent Systems and Applications III, Springer, Berlin Heidelberg, 2003.

[19] Gustafsson, A., A. Herrmann, and F. Huber, Conjoint Measurement: Methods and Applications, Springer, Berlin New York, 2007.

[20] Helbig, M., K. Deb, and A. Engelbrecht, "Key challenges and future directions of dynamic multi-objective optimisation", in IEEE Congress on Evolutionary Computation (CEC), Vancouver, BC, Canada, 2016.

[21] Jacquet-Lagreze, E. and J. Siskos, "Assessing a set of additive utility functions for multicriteria decision-making, the UTA method", European Journal of Operational Research, 10(2), 1982, pp. 151-164.

[22] Johnson, R.M., "Adaptive Conjoint Analysis", in Sawtooth Software Conference Proceedings, Sawtooth Software, Ketchum, ID, 1987.

[23] Keeney, R.L. and H. Raiffa, Decisions with multiple objectives: Preferences and value tradeoffs, Wiley, New York, 1976.

[24] Kersten, G.E., S. Szpakowicz, and Z. Koperczak, "Modelling of decision making for discrete processes in dynamic environments", Computers \& Mathematics with Applications, 20(9-10), 1990, pp. 29-43.

[25] Lai, S.-K., "A preference-based interpretation of AHP", Omega, 23(4), 1995, pp. 453-462.

[26] Lenz, A., "Supporting Decisions in Requirements Negotiations Dynamically", in Softwaretechnik-Trends, Gesellschaft für Informatik, 38(1), 2018.

[27] Lenz, A. and M. Schoop, "Decision Problems in Requirements Negotiations - Identifying the Underlying Structures", in Group Decision and Negotiation. A SocioTechnical Perspective, Stuttgart, Germany, 2017, Springer International Publishing, Cham. 2017, pp. 120-131

[28] Liechty, J.C., D.K.H. Fong, and W.S. DeSarbo, "Dynamic Models Incorporating Individual Heterogeneity: Utility Evolution in Conjoint Analysis", Marketing Science, 24(2), 2005, pp. 285-293.

[29] Matzner, M., M. von Hoffen, T. Heide, F. Plenter, and F. Chasin, "A Method for Measuring User Preferences in Information Systems Design Choices", in ECIS 2015 Completed Research Papers, 2015.

[30] Meißner, M., R. Decker, and N. Adam, "Ein empirischer Validitätsvergleich zwischen Adaptive Self-
Explicated Approach (ASE), Pairwise Comparison-based Preference Measurement (PCPM) und Adaptive Conjoint Analysis (ACA)", Zeitschrift für Betriebswirtschaft, 81(4), 2011, pp. 423-446.

[31] Nebro, A.J., A.B. Ruiz, C. Barba-González, J. GarcíaNieto, M. Luque, and J.F. Aldana-Montes, "InDM2: Interactive Dynamic Multi-Objective Decision Making Using Evolutionary Algorithms", Swarm and Evolutionary Computation, 2018.

[32] Netzer, O. and V. Srinivasan, "Adaptive SelfExplication of Multiattribute Preferences", Journal of Marketing Research, 48(1), 2011, pp. 140-156.

[33] Netzer, O., O. Toubia, E.T. Bradlow, E. Dahan, T. Evgeniou, F.M. Feinberg, E.M. Feit, S.K. Hui, J. Johnson, J.C. Liechty, J.B. Orlin, and V.R. Rao, "Beyond conjoint analysis: Advances in preference measurement", Marketing Letters, 19(3-4), 2008, pp. 337-354.

[34] Raiffa, H., J. Richardson, and D. Metcalfe, Negotiation analysis: The science and art of collaborative decision making, Belknap Press of Harvard University Press, Cambridge, MA, 2002.

[35] Reiser, A., Entscheidungsunterstützung in elektronischen Verhandlungen: Eine Analyse unter besonderer Berücksichtigung von unvollständigen Informationen, Springer Gabler, Wiesbaden, 2013.

[36] Roszkowska, E. and T. Wachowicz, "Application of fuzzy TOPSIS to scoring the negotiation offers in illstructured negotiation problems", European Journal of Operational Research, 242(3), 2015, pp. 920-932.

[37] Roy, B., "The Outranking Approach and the Foundations of Electre Methods", in Readings in Multiple Criteria Decision Aid, Springer, Berlin Heidelberg, 1990.

[38] Saaty, T.L., "How to make a decision: The analytic hierarchy process", European Journal of Operational Research, 48(1), 1990, pp. 9-26.

[39] Schlereth, C., C. Eckert, R. Schaaf, and B. Skiera, "Measurement of preferences with self-explicated approaches: A classification and merge of trade-off- and non-trade-off-based evaluation types", European Journal of Operational Research, 238(1), 2014, pp. 185-198.

[40] Scholz, S.W., M. Meissner, and R. Decker, "Measuring Consumer Preferences for Complex Products: A Compositional Approach Based on Paired Comparisons", Journal of Marketing Research, 47(4), 2010, pp. 685-698.

[41] Srinivasan, V. and C.S. Park, "Surprising Robustness of the Self-Explicated Approach to Customer Preference Structure Measurement", Journal of Marketing Research, 34(2), 1997, p. 286.

[42] Toubia, O., D.I. Simester, J.R. Hauser, and E. Dahan, "Fast Polyhedral Adaptive Conjoint Estimation", Marketing Science, 22(3), 2003, pp. 273-303.

[43] Vetschera, R., "Preference structures and negotiator behavior in electronic negotiations", Decision Support Systems, 44(1), 2007, pp. 135-146. 